\title{
Reliability analysis of observational data: Problems, solutions, and software implementation
}

\author{
ROLAND G. JANSEN, LEON F. WIERTZ, ERIK S. MEYER, and LUCAS P. J. J. NOLDUS \\ Noldus Information Technology, Wageningen, The Netherlands
}

\begin{abstract}
In the reliability analysis literature, little attention has been given to the various possible ways of creating a basis for the comparison required to compute observer agreement. One needs this comparison to turn a sequential list of behavioral records into a confusion matrix. It is shown that the way to do this depends on the research question one needs to answer. Four methods for creating a basis for comparison for the computation of observer agreement in observational data are presented. Guidelines are given for computing observer agreement in a way that fits one's goals. Finally, we discuss how these methods have been implemented in The Observer software. The Observer 4.1 supports all the methods that have been discussed. Most of these methods are not present in any other software package.
\end{abstract}

In observational research, reliability of data refers to the degree of agreement between sets of observational data collected independently from the same scene by two different observers (interobserver agreement) or by the same observer at different times in the data collection process (intraobserver agreement). Reliable data are a first prerequisite for answering research questions. It is important to determine whether data sets that are collected by different observers or at different times differ so little that one can safely assume that they are equally valid for analysis. Reliability analysis is also used to train new observers in coding schemes and observational data collection-for example, by testing their data against a reference data set, both collected from the same video recording.

Various quantitative measures have been used by researchers for the assessment of the degree of agreement between observers. Most commonly used measures for the consistency of data sets are Cohen's kappa (Cohen, 1960) for nominal data, Pearson's product moment correlation coefficient, commonly known as Pearson's $r$ (Bravais, 1846; Galton, 1888; Pearson, 1920), for interval data, and percentage of agreement and index of concordance when there is no measurement scale. The latter two are merely different expressions of the same quantity. We will not discuss the computational formulas for these measures; we assume that the readers are familiar with these. If not, please refer to statistical textbooks or the Internet.

The authors thank R. H. Geuze (University of Groningen, Groningen, The Netherlands) for his valuable input to and constructive comments on this paper. Correspondence should be addressed to R. G. Jansen, Noldus Information Technology bv, Costerweg 5, P .O. Box 268, 6700 AG Wageningen, The Netherlands (e-mail: r.jansen@noldus.nl).

- Note: The authors have a commercial interest in the software described in this paper.
Cohen's kappa is, in general, the measure of choice (Hollenbeck, 1978). Percentage of agreement does not take into account the rate of chance agreement, the base level of agreement under the assumption that each instance of behavior was linked at random to a behavioral category (throughout this paper, we will use behavioral category instead of behavioral element, which is less generic). This is especially important if one has few behavioral categories, because the fewer the categories, the bigger the chance that two raters will pick the same category, even if they pick the categories randomly (Cohen, 1960).

Pearson's correlation applies only to interval data, for which (formally) normality is assumed. However, an ethogram can generate nominal data as well as interval data, as we will show later. Note that one preserves more information when nominal data is generated than when interval data is generated.

Many programs have been developed that are capable of computing quantitative reliability measures. In fact, there are too many to mention, so we will mention just some of the authors, in chronological order, who have developed programs that are more or less specific to observational data: Watkins and Larimer, 1980; Burns and Cavallaro, 1982; Bloor, 1983; Chan, 1987; Repp, Harman, Felce, van Acker, and Karsh, 1989; Strube, 1989; Valiquette, Lesage, Cyr, and Toupin, 1994; Robinson and Bakeman, 1998. What these programs have in common is that confusion matrices (also known as association matrices or correlation matrices) are used as the starting point for the computations. Confusion matrices are contingency tables in which agreements and disagreements between two observers in the assessment of instances of behavior have been tallied. However, as we will demonstrate below, computing a reliability measure is not really a problem once you have the correct formula; transforming the observational data into a confusion matrix is the real issue. 
Table 1

Assigning a Nominal Value or Label (A to D) to Instances of Behavior (Cases)

\begin{tabular}{ccc}
\hline Case & Observer 1 & Observer 2 \\
\hline 1 & A & A \\
2 & C & B \\
3 & D & D \\
4 & A & C \\
5 & C & C \\
6 & B & B \\
7 & D & D \\
\hline
\end{tabular}

Table 2

Tallying Instances of Behavior for Each Nominal Category (Case), Thus Creating Interval Data

\begin{tabular}{ccc}
\hline & \multicolumn{2}{c}{ Sum of Behavioral Instances } \\
\cline { 2 - 3 } Case & Observer 1 & Observer 2 \\
\hline A & 2 & 1 \\
B & 1 & 2 \\
C & 2 & 2 \\
D & 2 & 2 \\
\hline
\end{tabular}

Some literature has addressed specific problems that arise in determining observer agreement because of the time-structured and multivariate nature of the observational data (Haccou \& Meelis, 1992; Hollenbeck, 1978; MacLean, Tapp, \& Johnson, 1985; Repp et al., 1989; Suen \& Ary, 1989; Thomann, 2001). However, no paper has listed all problems or provided for a full and thorough solution to the problems addressed.

In this paper, we will discuss some methodological problems related to the assessment of observer agreement on observational data, how these can be solved, and how these solutions have been implemented with The Observer software. The Observer is a software package for the collection, management, analysis, and presentation of observational data (Noldus, 1991; Noldus, Trienes, Hendriksen, Jansen, \& Jansen, 2000). One can use it to record activities, postures, movements, positions, facial expressions, social interactions, or any other aspect of human or animal behavior.

Version 5.0, the latest release at the time of writing, contains a new reliability analysis module with several new functions that will be discussed below. Note that in this paper, observer agreement will be used to denote both interobserver and intraobserver agreement.

\section{COMPUTING OBSERVER AGREEMENT FOR OBSERVATIONAL DATA}

The following three problems with respect to the computation of observer agreement for observational data will be discussed below: what cases should be compared, how to analyze at the right level of precision, and how to determine for which behavioral categories observers agreed the least.

\section{What Cases Should be Compared?}

Behavioral instances versus behavioral categories. In general, all measures that are used to assess observer agreement on observational data have been developed to assess the degree of correlation between two independent measurements of a number of independent cases. Cohen's kappa, for instance, was originally developed to assess the degree of agreement between two medical professionals in the diagnosis of the conditions from which patients suffer (Cohen, 1960).

In Cohen's (1960) original setting, each patient represented a case, and each medical condition was a value to be assigned to a case. However, in observational research, it is not clearly defined what the cases and the possible values are. Consequently, one cannot readily interpret any kappa value (or other reliability measure) as long as one does not know exactly which data were used to compute kappa.

We propose two approaches to identifying cases. (1) A case is represented by an instance of behavior, and the behavioral categories represent the possible values (i.e., measurement is done on a nominal scale). One then approaches the process of recording behavior as labeling instances of behavior - that is, linking a behavioral category to each instance of behavior (Table 1). The order of cases equals the chronology of the observed process. (2) A case is represented by a behavioral category, and the sum of the behavioral instances for each behavioral category within the data set represents the values (i.e., measurement is done on an interval scale). The behavior

Table 3

Frequency-Based Comparison: Each Behavior Scored Equals a Case

\begin{tabular}{ccc}
\hline Case & Observer 1 & Observer 2 \\
\hline 1 & A & A \\
2 & C & B \\
3 & D & D \\
4 & A & C \\
5 & C & C \\
6 & B & B \\
7 & D & D \\
\hline
\end{tabular}

Table 4

Duration-Based Comparison: Each Unit of Time Equals a Case

\begin{tabular}{ccc}
\hline Case & Observer 1 & Observer 2 \\
\hline 1 & A & A \\
2 & $\mathrm{C}$ & $\mathrm{A}$ \\
3 & $\mathrm{C}$ & $\mathrm{B}$ \\
4 & $\mathrm{D}$ & $\mathrm{D}$ \\
5 & $\mathrm{D}$ & $\mathrm{C}$ \\
6 & $\mathrm{~A}$ & $\mathrm{C}$ \\
7 & $\mathrm{C}$ & $\mathrm{C}$ \\
8 & $\mathrm{C}$ & $\mathrm{C}$ \\
9 & $\mathrm{C}$ & $\mathrm{C}$ \\
10 & $\mathrm{~B}$ & $\mathrm{C}$ \\
11 & $\mathrm{D}$ & $\mathrm{B}$ \\
12 & $\mathrm{D}$ & $\mathrm{D}$ \\
\hline
\end{tabular}


Table 5

Example of Two Streams of Data From the Same Scene Recorded by Different Observers

\begin{tabular}{ccccc}
\hline \multicolumn{2}{c}{ Observer 1} & & \multicolumn{2}{c}{ Observer 2} \\
\cline { 2 - 2 } \cline { 5 - 5 } Time & Behavior & & Time & Behavior \\
\hline 0 & walk & & 0 & walk \\
3 & jog & & 5 & run \\
8 & hold & & 8 & hold \\
10 & walk & & 12 & run \\
13 & run & 16 & walk \\
17 & \{end & 17 & end \\
\hline
\end{tabular}

Table 6

Tallying Instances of Behavior With a

Duration-Based Comparison

\begin{tabular}{lcc}
\multicolumn{3}{c}{ Duration-Based Comparison } \\
\hline Case & Observer 1 & Observer 2 \\
\hline Jog & 5 & 0 \\
Walk & 6 & 6 \\
Run & 4 & 7 \\
Hold & 2 & 4 \\
\hline
\end{tabular}

recording process is regarded as tallying instances of behavior for a number of behavioral categories (Table 2). The chronology of the observed process cannot be deduced from this table, since there is no temporal order in the list of cases.

In theory, these different approaches could be reflected by different data-logging methods. It is clear that Table 1 contains nominal data, whereas Table 2 contains interval data. However, there have been two developments in the past 15 years that have caused a high degree of standardization in data logging. First of all, the introduction of computer-aided recording of behavior has resulted in a major shift toward logging behaviors as nominal data, even if logging them as interval data would have sufficed to answer the research questions. This is because the computer can record the time stamp automatically and can easily turn the sequential data of the kind presented in Table 1 into the contingency table presented in Table 2 . In other words, one can easily transform nominal data into interval data, but not the other way round, so it is always safer to log behaviors as nominal data. Second, thanks to the increased availability and ease of use of applications that allow the coding of video at single-frame accuracy (Noldus et al., 2000), researchers more and more use continuous sampling, in- stead of time sampling (interval sampling). The philosophy behind this development is simple: One gets more information with hardly any extra effort. In continuous sampling, instances of observational data are recorded continuously, which results in the recording of the same instance of behavior at different times for different observers. This makes it difficult to tell which behavioral records from the observers refer to the same instance of behavior, which is no problem at all when time sampling is used. Moreover, since observers may overlook behaviors, accidentally enter a behavior, or record a behavior that they think they saw but that did not occur, just determining the number of instances of behavior is difficult. Because researchers may use continuous sampling when interval sampling, or even old-fashioned tallying of behaviors per category, would have sufficed, one cannot decide upon an approach to reliability analysis on the basis of the data recording method only, and one must take research goals into consideration.

One chooses the labeling instances of behavior approach if the focus is on qualitative analysis of data-in other words, if it is important that the observers agree on the assessment of each specific instance of behavior (e.g., because one is interested in the temporal and sequential analysis of the data). For instance, in usability studies, the human observer may need to know the exact sequence of events that causes the test participant to make an error in the operation of the computer interface that is being studied. Or, in the study of mother-child interactions, one may be interested in the presence and significance of particular sequential patterns of behaviors, and one thus needs an accurate registration of the sequence of events.

In contrast, the tallying instances of behavior approach is simpler to apply and does not require linking of specific behavioral records. One chooses this approach if the focus is on quantitative analysis of data-in other words, if it is important that the observers agree on the number of behavioral instances for each behavioral category and the timing and sequence of behavioral instances are not of interest. For instance, if one needs to answer a research question such as "Is there more shouting in the classroom during biology class than during math class?" it is not important who does the shouting, when during the course of the class the shouting is done, or what else is happening in the classroom. Just the count of instances of shouting matters.

Table 7

Confusion Matrix Resulting From the Data of Table 6

\begin{tabular}{lcccccr}
\hline & \multicolumn{6}{c}{ Observer 1 } \\
\cline { 2 - 7 } Observer 2 & Jog & Walk & Run & Hold & Disagreement & Total \\
\hline Jog & 0 & - & - & - & 0 & 0 \\
Walk & - & 6 & - & - & 0 & 6 \\
Run & - & - & 4 & - & 3 & 7 \\
Hold & - & - & - & 2 & 2 & 4 \\
Disagreement & 5 & 0 & 0 & 0 & - & 5 \\
Total & 5 & 6 & 4 & 2 & 5 & 22 \\
\hline
\end{tabular}


Table 8

Tallying Instances of Behavior With a Frequency-Based Comparison

\begin{tabular}{lcc}
\hline Case & Observer 1 & Observer 2 \\
\hline Jog & 1 & 0 \\
Walk & 2 & 2 \\
Run & 1 & 2 \\
Hold & 1 & 1 \\
\hline
\end{tabular}

Both approaches to reliability analysis have been discussed briefly in the literature. For example, Johnson and Bolstad (1973) noted that when you compute observer agreement on the basis of the sum of the instances of behavior per behavioral category per data set, high observer agreement might be misleading because this agreement may have been based on different instances of behavior. This is indeed true when one is interested in the evaluation of sequence, timing, or duration of the recorded behaviors. We, however, want to point out that if, for analysis, one is not interested in the sequence, timing, and duration of instances of behaviors, but only in overall statistics (count, total duration, or mean duration) for the complete observation, this method is a valid and preferable alternative, because it better fits the analysis goal. Still, it is important that one should always look at the marginal totals in the confusion matrix, and not just at the correlation coefficient, because Pearson's $r$ remains unaffected if one observer consistently overestimates the number of instances of behavior for each behavioral category by a certain factor. For instance, if one observer has exactly twice as many tallies for each behavioral category, as compared with a second observer, Pearson's $r$ is the same for both observers.

Frequencies of behavioral instances versus durations of behavioral instances. Until now, we have used the phrase instance of behavior. However, we have not defined it exactly, because we felt that there were two valid definitions here, too, depending on one's research questions. One could simply state that an instance of behavior is the occurrence of (the onset of) a behavioral event (to be referred to as the frequency-basedapproach; see Table 3), or one could state that it is a unit of time (e.g., $1 \mathrm{sec}$ ) for which behavior must be recorded (to be referred to as the duration-based approach; Table 4).

The interpretation of the phrase instance of behavior has a big impact on the way one does reliability analysis.
If one defines an instance of behavior as a behavioral event, one has far fewer cases for comparison. Using unit of time as one's definition of an instance of behavior is actually a way to take the duration of the behavioral events into account for reliability analysis, because behavioral events that last longer will result in more instances of behavior and will have more impact on the reliability scores.

The frequency-based and duration-based approaches have been discussed in the literature. Hollenbeck (1978) wrote that for duration-based comparison (or second-bysecond comparison, as he named it), it is arguable whether observers actually make a decision about a behavior continuously or only as a change in behavior occurs. MacLean et al. (1985) did not engage in much discussion on this. They simply stated that, for event data without duration information, one should choose the frequency-based approach and, for event data with duration information, one should choose the duration-based approach.

In our opinion, the authors of both papers passed over what reliability analysis is all about: verifying whether one's data can be trusted to give a fair answer to the research questions. So, by definition, how one does reliability analysis depends on what research questions one needs to answer eventually: If the question is, "Do people eat more cookies when they eat together than when they are alone?" then it is clearly related to frequencies of behavior, and consequently, a frequency-based approach to reliability analysis should be chosen. If, however, the question is "Do people spend a longer time eating when they are together than when they are alone?" then it is related to the durations of behavior, and a duration-based approach to reliability analysis should be chosen. Note that if one needs to answer both research questions, one should do two separate reliability analyses in support of the answers.

Four methods for identifying cases for comparison. The approaches to the problem of identifying the cases for comparison, described in the previous two sections, result in four methods for identifying cases for comparison.

Tallying instances of behavior with a duration-based comparison (Tables 5, 6, and 7). For each behavioral category, the total duration of instances is computed. If a study were designed to answer a question like "Does the object of study walk for a higher percentage of time in the first situation than in the second?" then this would be

Table 9

Confusion Matrix Resulting From the Data of Table 8

\begin{tabular}{lcccccc}
\hline & \multicolumn{7}{c}{ Observer 1 } \\
\cline { 2 - 7 } Observer 2 & Jog & Walk & Run & Hold & Disagreement & Total \\
\hline Jog & 0 & - & - & - & 0 & 0 \\
Walk & - & 2 & - & - & 0 & 2 \\
Run & - & - & 1 & - & 1 & 2 \\
Hold & - & - & - & 1 & 0 & 1 \\
Disagreement & 1 & 0 & 0 & 0 & - & 6 \\
Total & 1 & 2 & 1 & 1 & 1 & 1 \\
\hline
\end{tabular}


Table 10

Labeling Instances of Behavior With a Duration-Based Comparison

\begin{tabular}{|c|c|c|c|c|}
\hline \multirow[b]{2}{*}{ Case } & \multicolumn{2}{|c|}{ Observer 1} & \multicolumn{2}{|c|}{ Observer 2} \\
\hline & Time & Behavior & Time & Behavior \\
\hline 1 & 0 & walk & 0 & walk \\
\hline 2 & 1 & walk & 1 & walk \\
\hline 3 & 2 & walk & 2 & walk \\
\hline 4 & 3 & jog & 3 & walk \\
\hline 5 & 4 & jog & 4 & walk \\
\hline 6 & 5 & jog & 5 & run \\
\hline 7 & 6 & jog & 6 & run \\
\hline 8 & 7 & jog & 7 & run \\
\hline 9 & 8 & hold & 8 & hold \\
\hline 10 & 9 & hold & 9 & hold \\
\hline 11 & 10 & walk & 10 & hold \\
\hline 12 & 11 & walk & 11 & hold \\
\hline 13 & 12 & walk & 12 & run \\
\hline 14 & 13 & run & 13 & run \\
\hline 15 & 14 & run & 14 & run \\
\hline 16 & 15 & run & 15 & run \\
\hline 17 & 16 & run & 16 & walk \\
\hline
\end{tabular}

Table 11

Confusion Matrix Resulting From the Data of Table 10

\begin{tabular}{lccccr}
\hline & \multicolumn{5}{c}{ Observer 1 } \\
\cline { 2 - 6 } Observer 2 & Jog & Walk & Run & Hold & Total \\
\hline Jog & 0 & 0 & 0 & 0 & 0 \\
Walk & 2 & 3 & 1 & 0 & 0 \\
Run & 3 & 1 & 3 & 0 & 7 \\
Hold & 0 & 2 & 0 & 2 & 4 \\
Total & 5 & 6 & 4 & 2 & 17 \\
\hline
\end{tabular}

the comparison method of choice, because the researchers need to verify only whether the observers were consistent in measuring the percentage of time walking.

Tallying instances of behaviors with a frequency-based comparison (Tables 5, 8, and 9). For each behavioral category, the number of instances is counted. If a study were designed to answer a question like "Does walk occur more often in the first situation than in the second?" then this would be the comparison method of choice, because the researchers need to verify only whether the observers were consistent in counting the bouts of walking. Most often, this approach is related to the study of point events.

Labeling instances of behavior with a duration-based comparison (Tables 5, 10, and 11). Behavioral instances are compared on a time-by-time basis and are linked directly to the instance recorded by the other observer at the same time. If a study were designed to answer a question like "Does the object of study talk more while walking when he or she is in the first situation than when he or she is in the second?" then this would be the right comparison method, because the researchers need to be sure that the timing of walk and talk bouts was accurate in order to compute the amount of time for which those behaviors occurred at the same time. This would also be the method of choice if the researchers wanted to know whether longer periods of hold occurred after jog bouts than after walk bouts, because the researchers then would need to verify whether the sequence and duration of walk, jog, and hold bouts were recorded accurately.

Labeling instances of behavior with a frequency-based comparison (Tables 5, 12, and 13). Each instance of behavior is linked to an instance of behavior recorded by the other observer. Which other instance an instance of behavior is linked to depends on its behavioral category, timing, and sequence. If researchers want to know the answer to a question like "Is there a particular pattern that often precedes a run bout?" then this will be the method of choice, because answering this question requires an accurate registration of the sequence of instances of behavior.

Again note that when researchers need to answer different questions, they may need to run separate reliability analysis runs and use multiple comparison methods.

Table 5 presents an example to which these methods have been applied (shown in Tables 6-13). Contrary to the previous examples, time stamps have been added to instances of behaviors to illustrate additional problems that are specific to continuously recorded data. Furthermore, the confusion matrices are shown. This illustrates that the resulting confusion matrices, which are used as the basis for computation of reliability measures, are different, depending on the comparison method.

Note that labeling instances of behavior with a frequency-based comparison (Table 12) is not as straightforward as it may sound. For instance, do jog (time = 3) and run (time $=5$ ) refer to the same behavioral event in the real world, or did both observers miss out on a behavioral event? Or more generally, when should two behavioral records be considered to refer to the same instance of behavior in the real world and when should they be considered to refer to separate instances of behavior?

This difficulty can be countered by applying a tolerance window that sets the limits for how far off the timing of behavioral records in either data set is allowed to

Table 12

Labeling Instances of Behavior With a Frequency-Based Comparison

\begin{tabular}{cccccc}
\hline & \multicolumn{2}{c}{ Observer 1 } & & \multicolumn{2}{c}{ Observer 2 } \\
\cline { 2 - 3 } \cline { 5 - 6 } Case & Time & Behavior & & Time & Behavior \\
\hline 1 & 0 & walk & & 0 & walk \\
2 & 3 & jog & & 3 & walk \\
3 & 3 & jog & & 5 & run \\
4 & 8 & hold & & 8 & hold \\
5 & 10 & walk & 12 & run \\
6 & 13 & run & 16 & walk \\
\hline
\end{tabular}

Table 13

Confusion Matrix Resulting From the Data of Table 12

\begin{tabular}{lccccc}
\hline & \multicolumn{5}{c}{ Observer 1 } \\
\cline { 2 - 6 } Observer 2 & Jog & Walk & Run & Hold & Total \\
\hline Jog & 0 & 0 & 0 & 0 & 0 \\
Walk & 1 & 1 & 1 & 0 & 3 \\
Run & 1 & 1 & 0 & 0 & 2 \\
Hold & 0 & 0 & 0 & 1 & 1 \\
Total & 2 & 2 & 1 & 1 & 6 \\
\hline
\end{tabular}


Table 14

Example of Two Streams of Data From the Same Scene Recorded by Different Observers

\begin{tabular}{ccccc}
\hline \multicolumn{2}{c}{ Observer 1} & & \multicolumn{2}{c}{ Observer 2} \\
\cline { 2 - 3 } Time & Behavior & & Time & Behavior \\
\hline 0 & walk & & 0 & walk \\
2 & jog & 3 & run \\
3 & leap & 5 & jump \\
6 & walk & 9 & walk \\
10 & hold & 10 & hold \\
\hline
\end{tabular}

be (Hollenbeck, 1978; MacLean et al., 1985; Repp et al., 1989). If this tolerance window is exceeded, one may conclude that the records refer to different instances of behavior. Of course, the optimal width of the tolerance window varies from situation to situation. Various factors may affect this width: the research question, the nature of the behavior involved (does it appear suddenly, or evolve slowly from another behavior), and so forth. The Observer 5.0 allows the user to define a tolerance window as part of the reliability analy sis settings.

However, what if Data Set 2 does not contain run (at time $=5$ ) but, rather, jog (at time $=6$ )? If the tolerance window is $2 \mathrm{sec}$, should one regard Observer 1's jog (time $=3$ ) and Observer 2's jog (time $=6$ ) as separate behavioral events in the real world, as seems intuitive when these are different codes, or do both records refer to the same instance of behavior, but one which the second observer simply entered too late? The difference is important because, if one makes the former assumption, the comparison will result in two disagreements, whereas if one makes the latter assumption, there will be just one disagreement.

In this example, it seems most probable that jog (time $=3$ ) and jog (time $=6)$ refer to the same instance of behavior: There are no other obvious candidates to link these records to, and, moreover, there is an overlap in time for these records from time $=6$ to time $=8$.

Still, had there been a record of run (time $=5)$ in the first data set, one would be inclined to link this record to jog (time $=6$ ), which is supposedly an error of judgment by one of the observers, and in addition, to conclude that the second observer missed out on an instance of behavior that corresponds with jog $($ time $=3$ ). This means that the interpretation of these records depends on other records. We think that this is exactly how it should work; after all, this method is about using sequential information to identify the instances of behavior.

Finally, another difficulty is illustrated (see Table 14). The two observers agree on walk (time $=0)$ and hold (time $=10$ ), but what about the other behavioral records? For instance, should leap (time $=3$ ) be linked to run $($ time $=3)$ and walk $($ time $=6)$ to jump $($ time $=5)$ ? Or should jog (time $=2$ ) be linked to run (time $=3$ ), leap (time $=3)$ to jump (time $=5)$, and walk $($ time $=6)$ to walk $($ time $=9)$ ? How should one set criteria for linking when multiple behavioral records are present, in either data set, between matching pairs?

As a solution to these linking problems The Observer 5.0 uses an algorithm that finds agreements by first linking behavioral records that overlap in time (these links are agreements per definition) and then linking identical records within the tolerance window. Then the algorithm tries to identify disagreements. It first links any still unlinked record to the nearest unlinked record in the other data set within the tolerance window. If there is none, it tries to link this record to any event within the tolerance window. And if that does not work, it will link this record to the nearest record in the other data set. This algorithm is a refinement of the algorithm described by Haccou and Meelis (1992). Its advantage is that it applies to all behavioral records, whether they contain states or point events. Moreover, the procedure is symmetric, meaning that it does not matter with which data set you start the comparison.

So, if the tolerance window were set to $2 \mathrm{sec}$, Table 14 would render the results presented in Table 15.

\section{How to Analyze at the Right Level of Precision}

The second major problem in reliability analysis of observational data is getting the right level of precision for computing observer agreement. Hollenbeck (1978) mentioned that both relatively broad units of behavior (molar behaviors), because of their ambiguous nature, and relatively minute units of behavior (molecular behaviors), because of their subtle differences, may give rise to unreliable recording of behavior, suggesting that there is an optimal granularity of the definition of units of behavior. So, the paradox in reliability analysis is that the more precisely one tries to record behavior, the more likely it is that one will get disagreements, and the lower one's values of reliability measures will become. This is especially true in observational research, since behavioral categories are of a composite nature and, typically, consist of a subject identifier, a behavior identifier, and possibly some behavior modifiers. Quite often, one does not need such a high level of precision for all research questions. The obvious remedy is that one combines (lumps) related elements at the analysis stage. If one does not do this, one will be doing an overly harsh assessment of reliability, which may lead one to think that the conclusions pertaining to one's research questions are invalid.

Table 15

Results of Comparison When Instances of Behavior (Drawn From Table 14) are Labeled With a Frequency-Based Comparison

\begin{tabular}{|c|c|c|c|c|}
\hline \multicolumn{2}{|c|}{ Observer 1} & \multicolumn{2}{|c|}{ Observer 2} & \multirow[b]{2}{*}{ Result } \\
\hline Time & Behavior & Time & Behavior & \\
\hline 0 & walk & 0 & walk & agreement \\
\hline 2 & jog & 3 & run & disagreement \\
\hline 3 & leap & 5 & jump & disagreement \\
\hline 6 & walk & 9 & walk & disagreement \\
\hline 10 & hold & 10 & hold & agreement \\
\hline
\end{tabular}




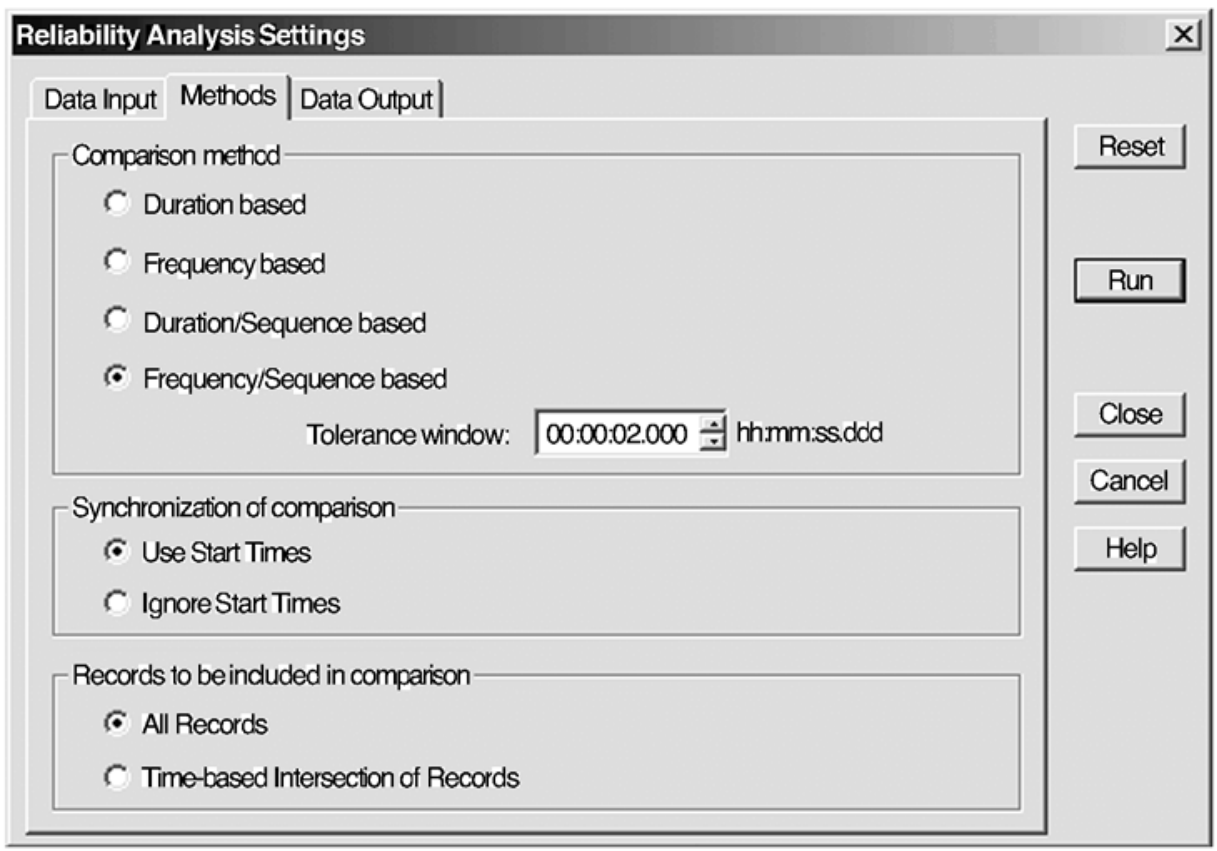

Figure 1. The Methods tab of the Reliability Analysis Settings dialog box of The Observer 5.0, showing all four comparison methods discussed in this paper, as well as the tolerance window option, which is specific for the frequency/sequence-based comparison method.

How to Determine for Which Behavioral Categories Observers Agreed the Least

Simply by looking at the literature references in this paper, one may conclude that much has been written about reliability measures. However, one overall value of a reliability measure does not provide one with any insight as to where exactly two observers failed to interpret the behavior the same way. Very little has been written about how one can find out for which behavioral categories observers agreed the least.

\begin{tabular}{|c|c|c|c|c|c|}
\hline \multicolumn{3}{|c|}{ Rocomotion - Reliability -Reliability Report (i) } & \multicolumn{3}{|c|}{$-10 x$} \\
\hline \multirow{2}{*}{ Result of Comparisons } & \multicolumn{2}{|c|}{ Observer 1} & \multicolumn{2}{|c|}{ Observer 2} & \multirow[t]{2}{*}{$\Delta$} \\
\hline & Time & Record & Time & Record & \\
\hline Agreement & $22-4-200311142145$ & John Walk & $22-4-200311142145$ & John Walk & \\
\hline Agreement & $22-4-200311142145$ & Sue Hold & $22-4-200311142145$ & Sue Hold & \\
\hline Disagreement & $22-4-200311142148$ & John Jog & $22-4-200311142150$ & John Run & \\
\hline Agreement & $22-4-200311142153$ & John Hold & $22-4-200311142153$ & John Hold & \\
\hline Disagreement & $22-4-200311142155$ & John Walk & $22-4-200311142153$ & John Hold & \\
\hline Agreement & $22-4-200311142158$ & John Run & $22-4-200311142157$ & John Run & \\
\hline Agreement & $22-4-200311143102$ & John Walk & 22-4-2003 11143101 & John Walk & \\
\hline Agreement & $22-4-200311143,13$ & John Jog & $22-4-200311143112$ & John Jog & \\
\hline Agreement & $22-4-200311143137$ & John Walk & $22-4-200311143134$ & John Walk & \\
\hline Agreement & $22-4-200311143139$ & John Hold & $22-4-200311143138$ & John Hold & \\
\hline Agreement & $22-4-200311,44,10$ & John Walk & $22-4-200311144,10$ & John Walk & \\
\hline Disagreement & $22-4-200311144,36$ & John Run & $22-4-200311144135$ & John Jog & \\
\hline Agreement & $22-4-200311,45100$ & John Walk & $22-4-200311145101$ & John Walk & \\
\hline Agreement & $22-4-200311145148$ & John Hold & $22-4-200311145146$ & John Hold & \\
\hline Agreement & $22-4-200311,45158$ & John Jog & $22-4-200311145157$ & John Jog & \\
\hline Agreement & $22-4-200311146111$ & John Walk & $22-4-200311146113$ & John Walk & \\
\hline Disagreement & $22-4-200311146,11$ & John Walk & $22-4-200311146136$ & John Jog & \\
\hline Agreement & $22-4-200311146112$ & SueWalk & $22-4-200311146111$ & Sue Walk & $\nabla$ \\
\hline $1 / \mathrm{K}$ Confusion Matrix & $\lambda$ List of Compariso & 1 & & & D? \\
\hline
\end{tabular}

Figure 2. An example of a list of compared behavioral records. Each row shows the two records that are linked and the result of comparison (agreement or disagreement). 


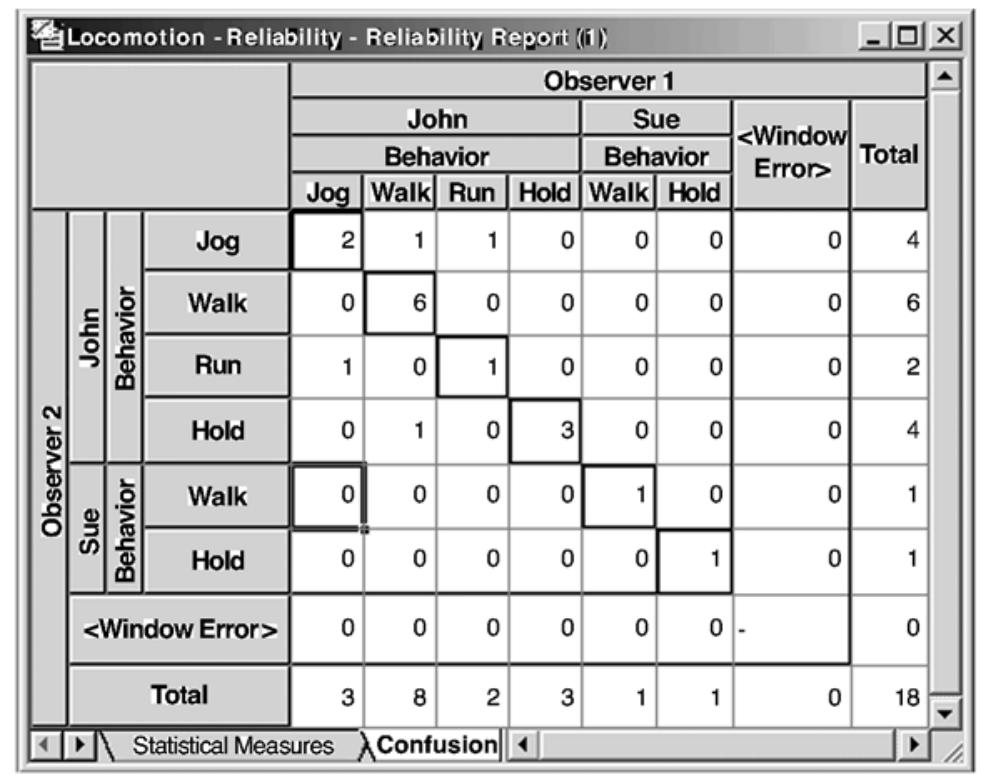

Figure 3. An example of a confusion matrix. The values on the diagonal represent the records that are in agreement. Off-diagonal values represent the disagreements.

Of course, one may take the straightforward approach described by van der Vlugt, Kruk, van Erp, and Geuze (1992). They evaluated where and when a trainee failed with a simple procedure in which the trainee and an experienced observer first independently annotated a videotape. They then replayed the videotape with a synchronized display of the recorded behaviors for both observers. However, this method is quite time consuming and is of no use for evaluating discrepancies once the data have been collected.

In order to understand the actual areas of disagreement, the confusion matrix is more useful than the values of the reliability measures. A particular value-for example, a kappa of 0.5 -does not tell one which behavioral categories disagree the most. One really needs to study the confusion matrix to find that out. Unfortunately, the topic of how to construct a confusion matrix has been completely overlooked in the reliability analysis literature, which traditionally has focused on the choice of the most suitable reliability measure and its computation. Indeed, it is fairly obvious how to construct a confusion matrix from data that are not time structured or data that are sampled at fixed intervals. However, it is far from obvious how to construct a confusion matrix from continuously recorded observational data. Such data typically consist of a sequential list of (nonequidistant) time-stamped behavioral records. Transformation from such a list into a confusion matrix (with intermediate results such as those presented in Tables 6-9) cannot be done in any data acquisition and analysis programs for observational research available so far, with the exception of GSEQ (Bakeman \& Quera, 1995). GSEQ, however, supports only the duration-based comparison method, in which instances of behavior are labeled, and none of the other comparison methods.

\section{RELIABILITY ANALYSIS IN THE OBSERVER 4.1}

The Observer 5.0 offers a new set of reliability functions that tackle all the problems discussed in the previous sections. Note that The Observer 3.0 already has supported one comparison method: labeling instances of behavior with a frequency-based comparison. However, back then, the method was implemented in a less sophisticated way.

The Observer 5.0 supports all four comparison methods, described above, that have been used to identify cases. These have been given the following names: duration-

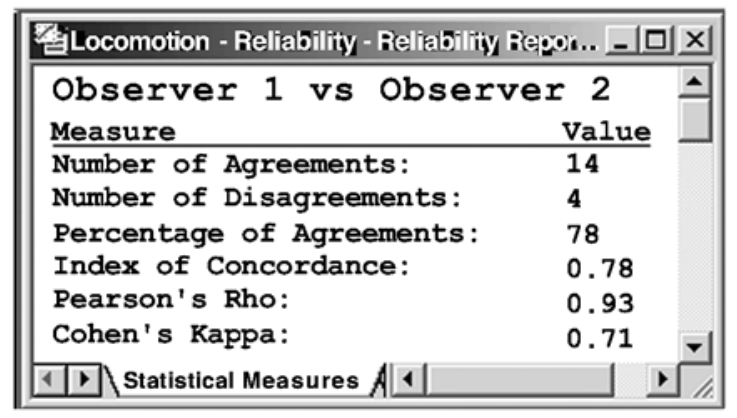

Figure 4. An example of the sheet in a reliability analysis report that shows the values computed for several reliability measures. 
based comparison, tallying instances of behavior with a duration-based comparison; frequency-based comparison, tallying instances of behaviors with a frequency-based comparison; duration/sequence-based comparison, labeling instances of behavior with a duration-based comparison; and frequency/sequence-based comparison, labeling instances of behavior with a frequency-based comparison. In addition, the tolerance window option and the advanced algorithms for labeling instances of behavior when frequency-based comparison is used are applied (Figure 1).

Also, one can tailor the precision of behavioral records to the level that is sufficient in relation to the research questions. This can be done by ignoring specific segments (subject, behavior, modifier) of each behavioral record or by grouping categories defined within segments.

Finally, outcomes are presented at different levels: a list of coupled records and their assessment (agreement or disagreement) for a detailed analysis of reliability per case (Figure 2), which list is shown only when instances of behavior are labeled with a frequency-based comparison; a confusion matrix that lists agreements on the diagonal and disagreements in off-diagonal cells (Figure 3); and an overview of reliability statistics, including percentage of agreement, index of concordance, Pearson's $r$, and Cohen's kappa (Figure 4).

\section{AVAILABILITY}

The Observer is commercially available from Noldus Information Technology and various international distributors. Readers can contact the first author for more information or visit the product homepage on the Internet (http://www.noldus.com/products/observer/).

\section{REFERENCES}

BAKeman, R, \& QUeRA, V. (1995). Analyzing interaction: Sequential analysis with SDIS and GSEQ. New York: Cambridge University Press.

BLOOR, R. N. (1983). A computer program to determine interrater reliability for dichotomous-ordinal rating scales. Behavior Research Methods \& Instrumentation, 15, 615 .

BravaIs, A. (1846). Analyse mathématique sur les probabilités des erreurs de situation d'un point. Mémoires présentés par divers savants à l'Académie royale des sciences de l'Institut de France, 9, 255-332.

Burns, E., \& Cavallaro,C. (1982). A computer program to determine interobserver reliability statistics. Behavior Research Methods \& Instrumentation, 14, 42.

Chan, T. S. C. (1987). A DBASE III program that performs significance testing for the Kappa coefficient. Behavior Research Methods, Instruments, \& Computers, 19, 53-54.
CoHEn, J. (1960). A coefficient of agreement for nominal scales. Educational \& Psychological Measurement, 20, 37-46.

Galton, F. (1888). Co-relations and their measurement, chiefly from anthropometric data. Proceedings of the Royal Society of London, 45, 135-145.

Haccou, P., \& Meelis, E. (1992). Statistical analysis of behavioural data: An approach based on time-structured models. Oxford: Oxford University Press.

Hollenbeck, A. R. (1978). Problems of reliability in observational data. In G. P. Sackett (Ed.), Observing behavior: Vol. 2. Data collection and analysis methods (pp. 79-98). Baltimore: University Park Press. Johnson, S. M., \& BolstaD, O. D. (1973). Methodological issues in naturalistic observation: Some problems and solutions for field research. In L. A. Hamerlynck, L. C. Handy, \& E. J. Mash (Eds.), Behavior change: Methodology, concepts and practice (pp. 7-67). Champaign, IL: Research Press.

MacLean, W. E., Tapp, J. T., \& Johnson, W. L. (1985). Alternate methods and software for calculating interobserver agreement for continuous observation data. Journal of Psychopathology \& Behavioral Assessment, 7, 65-73.

Noldus, L. P. J. J. (1991). The Observer: A software system for collection and analysis of observational data. Behavior Research Methods, Instruments, \& Computers, 23, 415-429.

Noldus, L. P. J. J., Trienes, R. J. H., Hendriksen, A. H. M., Jansen, H, \& JANSEN, R. G. (2000). The Observer Video-Pro: New software for the collection, management, and presentation of time-structured data from videotapes and digital media files. Behavior Research Methods, Instruments, \& Computers, 32, 197-206.

Pearson, K. (1920). Notes on the history of correlation. Biometrika, 13, 25-45.

Repp, A. C., Harman, M. L., Felce, D., van Acker, R., \& Karsh, K. G (1989). Conducting behavioral assessments on computer-collected data. Behavioral Assessment, 11, 249-268.

Robinson, B. F., \& BAKeman, R. (1998). ComKappa: A Windows '95 program for calculating kappa and related statistics. Behavior Research Methods, Instruments, \& Computers, 30, 731-734.

Strube, M. J. (1989). A general program for the calculation of the kappa coefficient. Behavior Research Methods, Instruments, \& Computers, 21, 643-644.

SuEN, H. K., \& ARY, D. (1989). Analyzing quantitative behavioral observation data. Hillsdale, NJ: Erlbaum.

Thomann, B. (2001). Observation and judgment in psychology: Assessing agreement among markings of behavioral events. Behavior Research Methods, Instruments, \& Computers, 33, 339-348.

Valiquette, C. A. M., Lesage, A. D., Cyr, M., \& Toupin, J. (1994). Computing Cohen's kappa coefficients using SPSS MATRIX. Behavior Research Methods, Instruments, \& Computers, 26, 60-61. VAN DER Vlugt, M. J., KRUK, M. R, vAN ERP, A. M. M., \& GeUZE, R. H (1992). CAMERA: A system for fast and reliable acquisition of multiple ethological records. Behavior Research Methods, Instruments, \& Computers, 24, 147-149.

WATKINS, M. W., \& LARIMER, L. D. (1980). Interrater agreement statistics with the microcomputer. Behavior Research Methods \& Instrumentation, 12, 466 .

(Manuscript received November 12, 2002; revision accepted for publication May 18, 2003.) 